\title{
Analysing the Behaviour and Performance of Opportunistic Routing Protocols in Highly Mobile Wireless Ad Hoc Networks
}

\author{
Varun G Menon ${ }^{1}$ and Joe Prathap P $\mathrm{M}^{2}$ \\ ${ }^{1}$ Research Scholar, Department of Computer Science Engineering \\ Sathyabama University, Chennai, India \\ varunmenon@scmsgroup.org \\ ${ }^{2}$ Professor, Department of Information Technology \\ RMD Engineering College, Chennai, India \\ drjoeprathap@rmd.ac.in
}

\begin{abstract}
Recent advances in wireless networks have enabled us to deploy and use mobile ad hoc networks for communication between the rescue officers in disaster recovery and reconstruction operations. This highly dynamic network does not require any infrastructure or centralized control. As the topology of the network remain dynamic, severe performance limitations incur with traditional routing strategies. Recently a new routing paradigm known as opportunistic routing protocols have been proposed to overcome these limitations and to provide efficient delivery of data in these highly dynamic ad hoc networks. Using the broadcasting nature of the wireless medium, this latest routing technique tries to address two major issues of varying link quality and unpredictable node mobility in ad hoc networks. Unlike conventional IP forwarding, where an intermediate node looks up a forwarding table for a suitable next hop, opportunistic routing brings in opportunistic data forwarding that allows multiple candidate nodes in the forwarding area to act on the broadcasted data packet. This increases the reliability of data delivery in the network with reduced delay. One of the most important issues that have not been studied so far is the varying performance of opportunistic routing protocols in wireless networks with highly mobile nodes. This research paper analyses and compares the various advantages, disadvantages and the performance of the latest opportunistic routing protocols in wireless ad hoc networks with highly mobile nodes.
\end{abstract}

Keyword-Ad hoc networks, Network Mobility, Opportunistic Routing, Performance Analysis, Reliability

\section{INTRODUCTION}

Mobile Ad hoc Network (MANET) is a group of nodes in a wireless communication network which dynamically forms temporary networks without the use of any existing infrastructure or centralized control. All the nodes are free to join or leave the network at any point of time. Most of the nodes in the network keep changing their position throughout. As the network is decentralized, the network organization and message delivery must be executed by the nodes themselves. Every node must be able to act as a host and also as a router to forward the packets for other mobile nodes. As the nodes change their position continuously entering and leaving the network, the topology of the network remains dynamic. The topology of MANETs does not remain static most of the time and keeps on changing with the movement of each mobile device in the network.

The popularity of MANETs has significantly increased over these years because of the numerous advantages created by its unique features like decentralized architecture, infrastructure-less design etc. This type of networking has its origin from the needs of communication between the soldiers in the military [1], and soon became very popular due to its applications in emergency situations, disaster relief operations and community networks. Some applications for common users can be connecting laptops for a live chat or conference session or using them in business meeting and conferences. Extensive research has been carried out in this field and a lot of research results have been published since the 1980's [2]. Due to this increasing interest for MANETs, over these years a lot of research have been carried out to move different applications from traditional infrastructure environment into the ad hoc context, so that a great deal of new services like multimedia streaming can be generated for the new environment. Although MANETs have brought in a lot of advantages to set up new applications, a number of issues still remain to be addressed. Unreliable wireless links and the dynamic network topology have been the two most important challenges faced in MANETs. Moreover in highly dynamic and fast changing MANETs, routing of packets with reliable and efficient data delivery remains an open challenge. Over these years a number of protocols have been proposed to solve this routing problem in wireless networks. Recent studies [3-8] shows that most of the routing protocols that follows traditional routing techniques like DSR [9-11], DSDV [12], AODV [13] and TORA [14-15] encounter numerous difficulties in handling with unpredictable wireless medium and random moving nodes. Most of these protocols relies on 
fixing a route before the data transmission and thus depends much more on the topology of the network. But in fast changing and reconfiguring MANETs, it's not possible to have a definite topology or definite route to send the packets. Once the path breaks, the data packet will be lost and considerable time and energy would be lost for the discovery and recovery procedures. So reliable and efficient delivery of data packets are not addressed by these protocols in fast changing mobile ad hoc networks.

One of the major contributions in this area was provided with the introduction of geographic routing [16-17]. Here the location information is used for routing the data packets from one node to the other node. The protocol makes use of Greedy forwarding to select next hop forwarder with the maximum positive progress towards the destination node. This protocol does not require any predetermined route for the transfer of the data packets which helps in better performance and scalability. But this routing strategy suffers from one major drawback that it is very much affected by incorrect information about the position of the nodes in the network [18-23]. The node which is at a maximum distance from the sender is chosen as the next hop. If the node moves out of the sender's coverage area, the transmission will fail. We observed this performance drawback using one of the most famous geographic routing protocols, Greedy Perimeter Routing protocol (GPSR) [24-25].

A major breakthrough in routing in mobile ad hoc networks was provided with opportunistic routing [3] and opportunistic data forwarding. Opportunistic routing makes use of the broadcasting property of the wireless networks to improve the efficiency of data delivery in the network. In wireless networks, when a data packet is send out, it is received by all other nodes within the transmission range on that channel. This property was often considered interference and a demerit of the wireless medium during these years. Opportunistic routing exploits the advantages brought about by this property. The concept of opportunistic routing [26-29] does not commit to a fixed route before data transmission. When a sender node wants to send a data packet to a particular destination device, it broadcasts the data packet into the network. This packet is received by every node within its transmission using MAC interception. The device that is closest to the destination is selected as the best forwarder device. This device forwards the data packet in a similar manner till it reaches the destination. Thus data forwarding remains dynamic and there is no need to maintain predetermined routes. Thus opportunistic routing provides an improved mechanism for the delivery of the data packet at the destination especially in ad hoc networks with highly mobile nodes. A number of opportunistic routing protocols have been proposed over these years. Every protocol has tried to address one or more issues involving data transfer in ad hoc networks. One of the most important issues that have not been studied so far is the varying performance of opportunistic routing protocols in wireless networks with highly mobile nodes. This paper analyses and compares the various advantages, disadvantages and the performance of the latest opportunistic routing protocols in wireless ad hoc networks with highly mobile nodes.

The rest of this article is organized as follows. Section 2 describes the working of opportunistic routing in detail. This section also describes the various routing metrics used by these protocols and also classifies the opportunistic routing protocols into five categories. All the major and latest applications using these routing protocols are described in this session. Section 3 describes the working of five most popular opportunistic protocols, one from each category. In section 4 results from simulations are used to analyse the behaviour and performance of these routing protocols in highly mobile ad hoc networks. In section 5 we conclude the article.

\section{Opportunistic Routing}

Opportunistic routing works with a very simple procedure. The source node broadcasts the data packet intended for any particular destination into the network. All the nodes in its transmission range receive the data packet through MAC interception [30]. These candidate nodes coordinate with each other and select one node to forward the data packet based on a Forwarder Priority List. The Forwarder Priority List (FPL) is developed with the help of metrics such as hop count, probability of loss, link loss probability, link delivery rate etc. The metric values are obtained based on current channel and propagation characteristics. Similarly the data packet is forwarded opportunistically from one node to the other node, till it reaches the destination. 


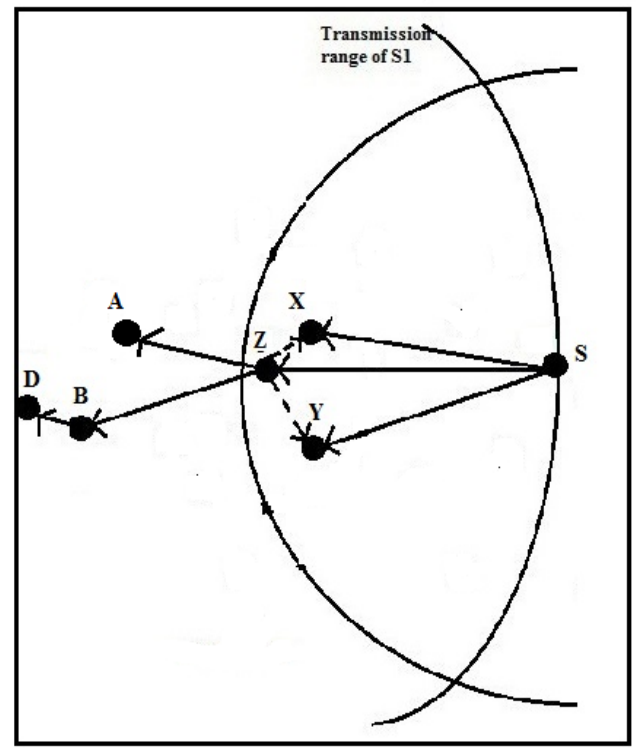

Fig. 1. Working of opportunistic routing protocol

Figure 1 depicts the working of opportunistic routing protocol in mobile ad hoc networks. Here the node $\mathrm{S}$ wants to send a data packet to the destination node D. Node $S$ broadcasts the data packet intended for the destination into the network. This data packet is received by nodes $\mathrm{X}, \mathrm{Y}$ and $\mathrm{Z}$ which are in the transmission range of node S. Based on the particular opportunistic routing strategy used; one node is selected as the forwarding node. Here we use the nearness to the destination as the deciding factor. So node $\mathrm{Z}$ which is the nearest to the destination is selected to forward the data packet. Node $\mathrm{Z}$ broadcasts the data packet into the network. Node $\mathrm{X}$ and node $\mathrm{Y}$ receives one copy of the data packet and thus understand that the data packet has already been forwarded by the best forwarder. So they discard the data packet. Node A and B also receives the data packet and based on the nearness o the destination node B is selected to forward the data packet to the destination. Node B checks for the destination in the list of neighbours and finds the destination D in it. So node B directly delivers the data packet to the destination node D.

\section{A) Different phases in Opportunistic Routing}

In order to better understand opportunistic routing, we can divide the entire routing process into four phases.

- $\quad$ Selection of the Candidate

- $\quad$ Priority Assignment for the Candidates

- $\quad$ Transmission of Data

- $\quad$ Coordination and Decision making

In the first phase of opportunistic routing a set of nodes are selected in the forwarding area of the source node .These nodes would act as potential forwarding nodes for a sender node and this list forms the forwarder list. In the next phase these forwarding nodes would be ordered and prioritized. In phase two, when a sender node informs about data transmission, each of these forwarding nodes are given different priorities based on metrics such as loss probability, number of hops to the destination etc. These metrics are specific to the particular opportunistic routing protocol used. In the third phase, the data is broadcasted by the source node into the network which is received by all the nodes in its transmission area. The last phase of opportunistic routing is the coordination of nodes in the forwarding list of the particular sender node. These nodes coordinate and select the best forwarder node based on certain metrics and channel characteristics for forwarding the data packet coming from the sender node. This strategy is repeated until the data packet reaches the destination mobile device.

\section{B) Opportunistic routing metrics}

The ordering of nodes in the forwarder list and prioritizing is based on some metrics that are specific to each opportunistic routing protocol.

- Anycast link cost: This metric is calculated using the transmission link properties such as delivery rate on the link. This metric uses the transmission link properties to order the forwarding nodes.

- Number of hops: Number of hops to the destination if also used as metric for ordering the nodes in the forwarder list. 
- Remaining path costs: This metric measures the properties of the remaining path to the destination for a forwarder node

- ETX (Expected transmission count). It is the number of tries required to successfully transmit a frame on a link

C) Applications

The opportunistic routing protocols have become much popular due to its numerous advantages and applications in various fields.

- Disaster recovery and Management: Opportunistic routing helps to establish reliable and continuous communication between the officers, medical team and other rescue workers in disaster recovery and emergency response situations [31-33]. Effective communication is very important in coordinating the rescue work and also in reconstruction works after the disaster. In order to carry out efficient and quick recovery, the rescue workers, police officers and everyone involved may have to move at a fast pace to different locations within the area to minimize the damage and to find out more survivors of the disaster. Opportunistic routing helps to deliver data between the rescue officers and helps in disaster recovery and reconstruction operations [34-35].

- Internet connectivity in remote areas: Opportunistic routing can be used to provide internet connectivity and data transfer in remote and rural areas. Ad hoc networks can be easily set up in these areas and opportunistic routing can be used for communication and data transfer. Opportunistic routing will also guarantee minimum quality of service in the network.

- Battlefield Communication: Opportunistic routing protocols guarantee reliable and continuous communication between soldiers in the battlefield and remote areas. This dynamic protocol can be used to send data between highly mobile soldiers in remote and infrastructure less areas during search and rescue operations.

D) Classification of Opportunistic Routing Protocols

We use the metric used for selecting and prioritizing the candidate nodes in the forwarder list as the reference to classify the opportunistic protocols into five categories.

- Link state aware Routing protocols: These routing protocols [3, 36-39] uses delivery rate over the transmission links to select and prioritize the forwarding node list.

- Hop count based routing protocols: These protocols [40-42] use the number of hops to the destination as the selection criteria for the forwarding nodes.

- Probabilistic opportunistic routing: These protocols [43-51] uses nodes encounter probabilities in forwarding in the network to generate the candidate list.

- Optimization based opportunistic routing protocols: These protocols [52-64] uses optimization techniques like graph based analysis to generate the candidate list.

- Location based opportunistic routing protocols: These routing protocols [31, 65-70] select candidate nodes for the forwarder list based on their location information.

\section{Opportunistic Routing Protocols}

We select the most popular and latest opportunistic routing protocols from each category for comparison and performance analysis. Thus we study about the most popular ExOR [3], OPRAH [40], EBR [48], LOR [60] and RRT [31] protocols in this section and lists down their advantages and disadvantages.

\section{A) Link state aware $\mathrm{OR}$}

One of the major objectives of opportunistic routing is to increase the rate of data delivery in the network. Opportunistic routing makes use of the broadcasting nature of the wireless medium to increase the number of potential forwarding candidates. This would ensure better data delivery and quality of service in the network. Link state aware protocols use the delivery probabilities in the wireless links to create the Candidate list. Extremely Opportunistic Routing (ExOR) is the first in this category of protocols and one of the most popular. ExOR was one of the first protocols that used the opportunistic forwarding mechanism in mobile ad hoc networks. ExOR introduced the batch mechanism in which the sender device will broadcast a batch of 10 to 100 packets. Each packet would contain a list of probable forwarding nodes. Every neighbour node in the list waits for its turn to forward the packet. Here the Forwarder Priority list is developed with ETX [3, 29] metric. ETX is the expected number of transmissions required to deliver a data packet. Inter-node loss rates are used to calculate the dynamic ETX values for various links in the network. The working of the protocol is divided into three phases, selecting the forwarding candidates, acknowledging transmissions, and deciding whether to forward a received. ExOR uses the minimum hop count and inter node loss rates with ETX to generate the priority list for each node in the network. Based on the list, the top priority node forwards the data packet. 
The major advantage of ExOR is that it has multiple paths for transmission and it increases the throughput considerably compared to traditional routing strategies. ExOR takes advantage of longer transmission to forward the packet further on each hop than predetermined routes which satisfy one of the primary objectives of opportunistic routing protocols. The major disadvantage of ExOR is that it uses a link state topology data base and flooding scheme which requires periodic network wide measurement which is very tough in highly mobile networks. Moreover communication and coordination between the candidate nodes generated duplicate transmissions when they were connected with transmissions links of low quality.

\section{B) Hop count based OR}

These protocols use the number of hops to the destination as the metric for generating the forwarder candidate list. OPRAH [40] is one of the most widely used protocols in this category. This protocol inherits multiple features of the AODV [13] protocol and discovers multiple paths to the destination. These routes are validated based on the number of hops in the particular route compared with the number of hops used in the optimum path so far. These protocols are less complex in design and work well in mobile ad hoc networks. The major advantage of OPRAH is that it is less complex and requires no MAC modification. The major disadvantage of this protocol is the inability to handle duplicate transmissions. Also using number of hops alone may not offer optimal path in highly dynamic ad hoc networks. Transmission and propagation characteristics of the link and the channel should be considered along with the number of hops to dynamically discover the best path to the destination.

\section{C) Probabilistic OR}

Due to high mobility of nodes in the network it is quite often difficult to set the right priority for the forwarding nodes. Generating candidate lists based on metrics like hop count, expected transmission count may not lead to optimal paths for the data packet. Using blind opportunistic forwarding for better performance may lead to enormous routing overhead. In order to improve the performance a new class of opportunistic routing protocols were found out which used various statistical measures and learning tools to identify the list of potential forwarders and to prioritize them. A number of Probabilistic Opportunistic Routing protocols [43-51] were proposed to improve the quality of service in highly mobile ad hoc networks. Encounter Based Routing, (EBR) [48] is of the popular and widely used protocol in this category. EBR protocol was proposed to decrease the number of duplicate transmissions in the network. EBR establishes an upper limit for the duplicate packets generated for transmission of a data packet in the network to limit the number of duplicate packets.EBR measures the number of encounters each node is having with the other nodes in the network. This encounter value forms the basis of assignment of priority for the forwarding nodes in the network. Nodes with more number of encounters are assumed to have better probability of data delivery at the destination node and are selected as the best forwarder over the other nodes. The major advantage of EBR is that it reduced the number of duplicate transmissions in the network and increased bandwidth and reduced the delay in transmissions. The major drawback of this protocol was that it could not increase the delivery rate in the network.

\section{D) Optimization based $O R$}

Optimization based opportunistic routing protocols tries to optimize the various parameters on which the opportunistic routing is build that includes the candidate list, forwarder selection and prioritization using game theoretic, machine learning and optimization programming approaches. Most of these protocols [52-64] use variations of graph theory to generate optimal results. Localized Opportunistic Routing (LOR) [60] is one of the widely used protocols in this category. The working of this protocol is based on graph theory. Using graph theory the entire network topology is partitioned into many smaller sub topologies. These smaller topologies are termed as close node sets. Now opportunistic routing is configured between these close node sets. Each close node set stores the local information and rearranges dynamically. The main advantages of this routing technique are its lower complexity and increased scalability compared to all previous approaches. The major drawback is that the protocol is sensitive to the local information for rearranging the close node sets and also this may increase the time and overhead in routing.

\section{E) Location based OR}

This category of opportunistic routing protocols uses location information for candidate list selection and decision making. A number of opportunistic protocols [31, 65-70] depend on the positional information of every node in the network for routing. Every time the position information needs to be updated. Reliable Routing Technique (RRT) [31] is one of the latest protocols using location information for routing in highly dynamic mobile ad hoc networks. This protocol is used to ensure reliable and continuous communication between the rescue workers in disaster recovery operations. Using RRT protocol when a node sends a data packet into the network, all the nodes in its transmission range receive the data packet. Then the forwarding area is determined for the source node. All the nodes in the forwarding area are selected to the candidate list. Priorities are assigned to each node based on its proximity to the destination. The node that is closer to the destination is given the first priority followed by the second. The first priority node is selected to forward the data packet. If that particular 
node moves away during this time, the next priority node forwards the data packet in the network. Thus communication is maintained, as long as one mobile device receives the transmission and thus the delivery of data packet at the destination is guaranteed with RRT. The main advantage with RRT is the high packet delivery ratio and reliability of data delivery in the network. Major area of concern in RRT is buffer occupancy. Table 1 shows the comparison of the protocols from the five categories.

TABLE I. Comparison of the Opportunistic Routing Protocols

\begin{tabular}{|c|c|c|c|c|c|c|}
\hline Protocol & Year & Classification & $\begin{array}{l}\text { Metric } \\
\text { Used }\end{array}$ & $\begin{array}{l}\text { Coordination } \\
\text { Method used }\end{array}$ & Advantages & Disadvantages \\
\hline ExOR & 2005 & $\begin{array}{c}\text { Link State Aware } \\
\text { OR }\end{array}$ & ETX & Timer + ACK & $\begin{array}{l}\text { Dynamic path } \\
\text { selection and } \\
\text { Increased } \\
\text { throughput }\end{array}$ & $\begin{array}{l}\text { Uses link state } \\
\text { style topology } \\
\text { database. }\end{array}$ \\
\hline OPRAH & 2006 & $\begin{array}{c}\text { Hop Count Based } \\
\text { OR }\end{array}$ & Hop count & Overhearing & $\begin{array}{c}\text { Less Complex } \\
\text { and no MAC } \\
\text { modification } \\
\text { required }\end{array}$ & $\begin{array}{c}\text { Not able to } \\
\text { handle duplicate } \\
\text { data } \\
\text { transmission }\end{array}$ \\
\hline EBR & 2009 & Probabilistic OR & $\begin{array}{l}\text { Delay } \\
\text { based }\end{array}$ & Overhearing & $\begin{array}{l}\text { Less number of } \\
\text { duplicate } \\
\text { transmission }\end{array}$ & $\begin{array}{c}\text { Stationary } \\
\text { delivery rate }\end{array}$ \\
\hline LOR & 2013 & $\begin{array}{l}\text { Optimization } \\
\text { Based OR }\end{array}$ & $\begin{array}{c}\text { Link } \\
\text { Quality }\end{array}$ & Overhearing & $\begin{array}{l}\text { Increased } \\
\text { scalability }\end{array}$ & $\begin{array}{l}\text { Sensitive to } \\
\text { local } \\
\text { information }\end{array}$ \\
\hline RRT & 2015 & $\begin{array}{c}\text { Location Based } \\
\text { OR }\end{array}$ & $\begin{array}{l}\text { Distance to } \\
\text { Destination }\end{array}$ & Timer & $\begin{array}{l}\text { Reliability of } \\
\text { data delivery }\end{array}$ & $\begin{array}{c}\text { Buffer } \\
\text { occupancy }\end{array}$ \\
\hline
\end{tabular}

\section{Results and Performance Analysis}

We use simulations in Network Simulator-2 [71] to study the performance of the five categories of opportunistic protocols. Initially we carry out simulation with 100 nodes in a uniformly distributed network topology. The packet size is set at 256 bytes and the transmission range is $250 \mathrm{~m}$. The nodes are distributed over a $1000 \mathrm{~m}$ $\times 800 \mathrm{~m}$ rectangular region. The two-ray ground propagation model is used for the simulation. Constant Bit Rate Traffic is generated between the nodes. The simulation time is set at 1000 seconds. In order to check the performance of these routing protocols in highly dynamic a hoc networks, the speed of the nodes is then varied from a minimum of $1 \mathrm{~m} / \mathrm{s}$ to various maximum limits in the topology setup to analyse the performance of the protocol in fast changing MANETs with highly mobile nodes. Mobility is introduced in the network with the Random Way Point mobility model [72]. Two important performance metrics, Packet Delivery Ratio and Average end to end delay are used to compare the performance of these protocols.

- Packet Delivery Ratio: It is defined as the ratio of data packets received at the destination(s) to the number of data packets sent by the source(s).

- $\quad$ Average end to end delay: The average delay in receiving an acknowledgement for a delivered data packet

Figure 2 shows the comparison of Packet Deliver Ratio's of the five different opportunistic protocols in highly mobile ad hoc networks. Packet Delivery Ratio (PDR) of ExOR which is a link state aware opportunistic protocol comes down a fraction when the mobility in the network increases. We have earlier seen that ExOR uses ETX as the decision making metric in generating the candidate list and selecting the best forwarder. PDR of OPRAH protocol that uses hop count as the decision making metric also comes down in a similar fraction. The delay based opportunistic protocol EBR maintains a slightly higher PDR compared to ExOR and OPRAH in highly dynamic ad hoc network. The optimization based opportunistic routing protocol, LOR maintains a better PDR compared to the previous three protocols. This shows that the link quality metric on which LOR is built works better compared to ETX, hop count and delay in fast changing mobile ad hoc networks. RRT which uses position information and distance to destination as the decision making metric has a PDR value close to 1 and maintains the performance even when the mobility of nodes increases in the network. This clearly shows that the opportunistic approaches using position information gives better data delivery in highly dynamic wireless ad hoc networks. 


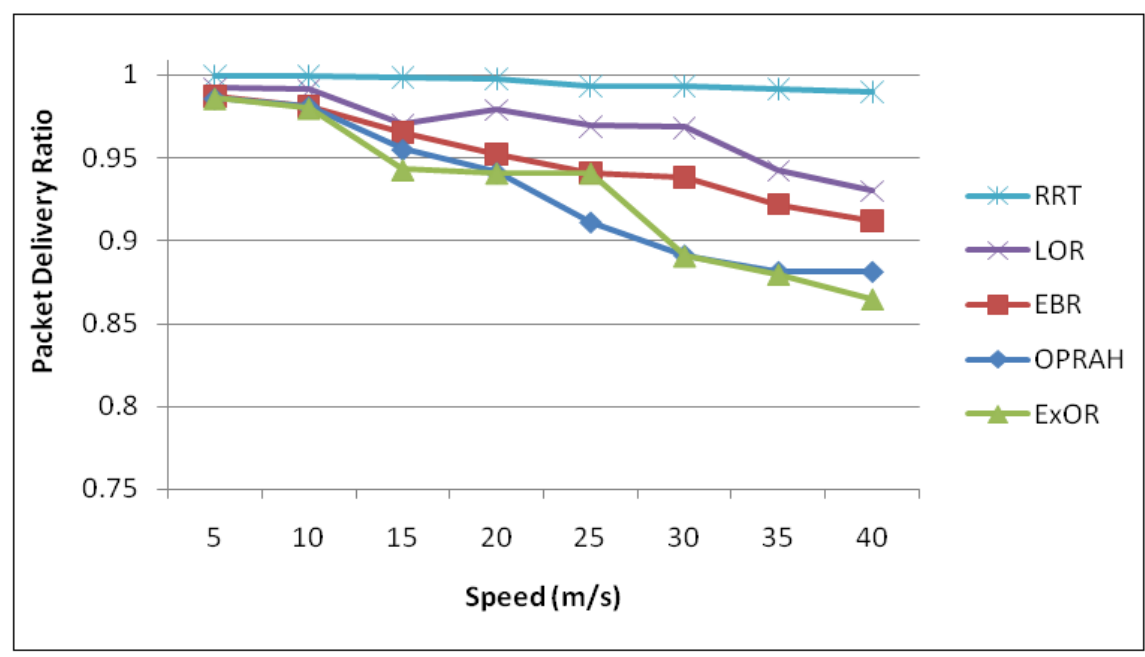

Fig. 2. PDR vs. Speed

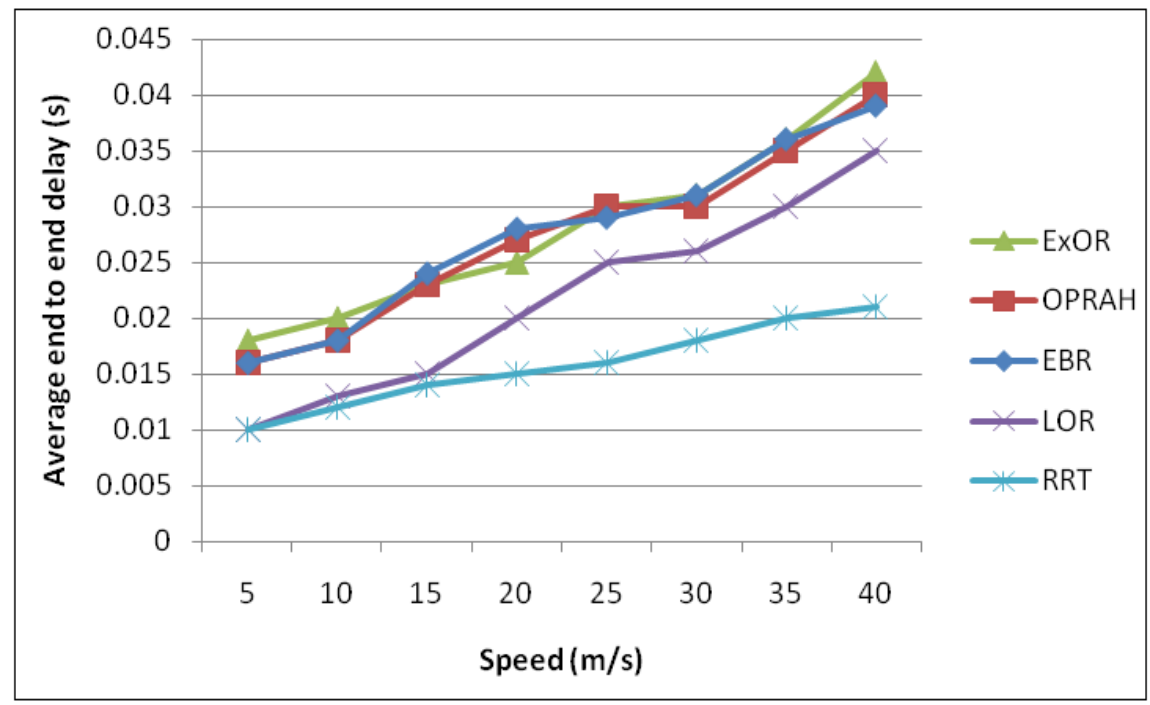

Fig. 3. Average end to end delay vs. Speed

Figure 3 shows the comparison of average end to end delays of the opportunistic protocols from the five categories in highly mobile ad hoc networks. Average end to end delay values of ExOR, OPRAH and EBR increases in a similar range as the mobility increases. The optimization based opportunistic protocol LOR has less delay in data transmission in the network compared to the previous three protocols. The average delay of RRT is much less compared to all the other opportunistic routing protocols.

This performance analysis clearly indicates that the opportunistic routing protocols working on link quality and positional information gives much better performance in highly dynamic mobile ad hoc networks. This information is vital for designing next level optimized opportunistic routing protocols that would guarantee very high performance even in highly mobile ad hoc networks.

\section{CONCLUSION}

In this paper we analysed the behaviour and performance of opportunistic routing protocols in highly mobile ad hoc networks. The working of opportunistic routing was discussed in detail along with the metrics used in each protocol. Latest applications of opportunistic routing that include maintaining communication in disaster recovery operations was discussed. We classified the opportunistic protocols into five categories and discussed the working of the most popular and widely used protocol from each category. Various advantages and disadvantages of these protocols were discussed in detail. We used simulations to analyse the performance of these protocols in highly mobile ad hoc networks. Results from simulations showed the variations in behaviour and performance of these opportunistic protocols. The performance analysis clearly showed that the opportunistic routing protocols working on link quality and positional information gives much better performance in fast changing MANETs. This study would further help in designing next level optimized opportunistic protocols that would guarantee very high quality of service in highly dynamic ad hoc networks. 


\section{REFERENCES}

[1] Barry M. Leiner, Robert J. Ruth, and Ambatipudi R. Sastry, “Goals and challenges of the DARPA GloMo program,” IEEE Personal Communications, 3(6):34-43, December 1996.

[2] I. Chlamtac, M. Conti, and J.-N. Liu, “Mobile Ad hoc Networking: Imperatives and Challenges,” Ad Hoc Networks, vol. 1, no. 1, pp. 13-64, July 2003.

[3] S. Biswas and R. Morris, "ExOR: opportunistic multi-hop routing for wireless networks," In Proc. of ACM SIGCOMM, Aug. 2005, .

[4] S. Chachulski, M. Jennings, S. Katti, and D. Katabi, "Trading structure for randomness in wireless opportunistic routing," In Proc. of ACM SIGCOMM, Aug. 2007.

[5] Shuai Wang , Basalamah, A. ,Song Min Kim, Shuo Guo ,Tobe, Y.; Tian He, "Link-Correlation-Aware Opportunistic Routing in Wireless Networks," in Wireless Communications, IEEE Transactions on, vol. 14, no. 1, pp. 47-56, Jan. 2015. .

[6] V. G. Menon and P. M. Joe Prathap, "Opportunistic routing with virtual coordinates to handle communication voids in mobile ad hoc networks," in Advances in Signal Processing and Intelligent Recognition Systems, vol. 425 of Advances in Intelligent Systems and Computing, pp. 323-334, Springer International, 2016.

[7] J. Broch, D.A. Maltz, D.B. Johnson, Y.-C. Hu, and J. Jetcheva, "a performance comparison of multi-hop wireless ad hoc network routing protocols,” Proc. ACM MobiCom, pp. 85-97, 1998.

[8] I. T. Haque, "On the overheads of ad hoc routing schemes," IEEE Systems Journal, Vol. 9, No. 2, pp. 605-614, June 2015.

[9] David B. Johnson. Routing in Ad Hoc Networks of Mobile Hosts. Proceedings of the Workshop on Mobile Computing Systems and Applications, pp. 158-163, IEEE Computer Society, Santa Cruz, CA, December 1994.

[10] D. Johnson and D. A Maltz, ”Dynamic Source Routing in A Hoc wireless networks”, Mobile Computing, pp.153-181, Kluwer Academic Publishers, 1996. .

[11] D. Johnson, Y. Hu, and D. Maltz, "The Dynamic Source Routing Protocol (DSR) for mobile ad hoc networks for IPv4," Internet Requests for Comments, RFC Editor, RFC 4728, Feb. 2007.

[12] C. E. Perkins and P. Bhagwat. "Highly dynamic destination-sequenced distance-vector routing (DSDV) for mobile computers", In Proc. of ACM SIGCOMM, 1994. .

[13] C. E. Perkins and E. M. Royer. "Ad hoc on-demand distance vector routing”, In Proc. of the 2nd IEEE Workshop on Mobile Computing Systems and Applications, Feb. 1999.

[14] Vincent D. Park and M. Scott Corson. "Applicability of the temporally-ordered routing algorithm for use in mobile tactical networks. Proceedings of IEEE Military Communications Conference, MILCOM, 1998.

[15] Vincent D. Park and M. Scott Corson. "A performance comparison of TORA and Ideal Link State routing," In Proceedings of IEEE Symposium on Computers and Communication '98, June 1998. .

[16] M. Mauve, A. Widmer, and H. Hartenstein, “A survey on position-based routing in mobile ad hoc networks,” IEEE Network, vol. 15, no. 6, pp. 30-39, Nov/Dec. 2001. .

[17] Fraser Cadger, Kevin Curran, Jose Santos, and Sandra Moffett, “A survey of geographical routing in wireless ad-hoc networks”, IEEE Communications Surveys \& Tutorials, Vol. 15, No. 2, July. 2013.

[18] D. Son, A. Helmy, and B. Krishnamachari, "The effect of mobility induced location errors on geographic routing in mobile ad hoc sensor networks: analysis and improvement using mobility prediction,” IEEE Trans. Mobile Computing, vol. 3, no. 3, pp. 233-23, Aug 2004. .

[19] S. Das, H. Pucha, and Y. Hu, "Performance Comparison of Scalable Location Services for Geographic Ad Hoc Routing," Proc. IEEE INFOCOM, vol. 2, pp. 1228-1239, Mar. 2005. .

[20] Y. Kim, J. Lee, and A. Helmy, "Impact of location inconsistencies on geographic routing in wireless networks," in Proc. ACM MSWIM'03, pp. 124-127, 2003.

[21] M. Zorzi and R. R. Rao, "Geographic random forwarding (GeRaF) for ad hoc and sensor networks: Multihop performance," IEEE Transactions in Mobile Computing., vol. 2, no. 4, pp. 337-348, Oct.-Dec, 2003 .

[22] Varun G. Menon and Joe Prathap P. M., Performance of various Routing Protocols in Mobile A Hoc Networks-A Survey, Research Journal of Applied Sciences, Engineering and Technology, 6(22), pp. 4181-4185, December 2013.

[23] Varun Menon and Joe Prathap P.M, "Performance analysis of geographic routing protocols in highly mobile ad hoc network," Journal of Theoretical and Applied Information Technology, vol. 54 no. 1, pp 127-133, 2013.

[24] B. Karp and H.T. Kung, “GPSR: Greedy Perimeter Stateless Routing for Wireless Networks,” Proc. ACM MobiCom, pp. 243-254, 2000.

[25] Varun G Menon, Jogi Priya P M, Joe Prathap P M, “Analyzing the behavior and performance of greedy perimeter stateless routing protocol in highly dynamic mobile ad hoc networks”, Life Science Journal , 10(2): pp 1601-1605, 2013.

[26] E. Rozner, J. Seshadri, Y. Mehta, and L. Qiu, "SOAR: simple opportunistic adaptive routing protocol for wireless mesh networks," IEEE Trans. Mobile Computing, vol. 8, no. 12, pp. 1622-1635, Dec. 2009. .

[27] Won-Yong Shin, Sae-Young Chung and Yong H. Lee,"Parallel opportunistic routing in wireless networks”, IEEE Transactions On Information Theory, Vol. 59, No. 10, pp6290-6300, October 2013. .

[28] Milen Nikolov and Zygmunt J. Haas, “Towards optimal broadcast in wireless networks," IEEE Transactions on Mobile Computing, Vol. 14, No. 7, July 2015.

[29] Shengbo Yang, Chai Kiat Yeo, and Bu Sung Lee, “Towards Reliable Data Delivery for Highly Dynamic Mobile Ad Hoc Networks”, IEEE Transactions On Mobile Computing, Vol. 11, No. 1, January 2012.

[30] N. Chakchouk, "A Survey on Opportunistic Routing in Wireless Communication Networks," in IEEE Communications Surveys \& Tutorials, vol. 17, no. 4, pp. 2214-2241, Fourth quarter 2015.

[31] Varun G. Menon, Joe Prathap Pathrose, and Jogi Priya, "Ensuring Reliable Communication in Disaster Recovery Operations with Reliable Routing Technique,” Mobile Information Systems, vol. 2016, Article ID 9141329, 10 pages, 2016.

[32] G. Gorbil and E. Gelenbe, "Resilient emergency evacuation using opportunistic communications," in Proc. 27th ISCIS, pp. 249-257, October 2012.

[33] A. Martin-Campillo, J. Crowcroft, E. Yoneki, and R. Marti, "Evaluating opportunistic networks in disaster scenarios," J. Netw. Comput. Appl., vol. 36, no. 2, pp. 870-880, Mar. 2013. .

[34] I. G. Askoxylakis, A. Makrogiannakis, A. Miaoudakis et al., "A rapid emergency deployment mobile communication node," in Proc. of the IEEE 19th International Workshop on Computer Aided Modeling and Design of Communication Links and Networks (CAMAD '14), pp. 290-294, Athens, Greece, December 2014.

[35] N. Pogkas, G. E. Karastergios, C. P. Antonopoulos, S. Koubias, and G. Papadopoulos, "Architecture design and implementation of an ad-hoc network for disaster relief operations,” IEEE Transactions on Industrial Informatics, vol. 3, no. 1, pp. 63-72, 2007.

[36] D. Koutsonikolas, C. Wang, and Y. Hu, "CCACK: Efficient network coding based opportunistic routing through cumulative coded acknowledgments,” in Proc. IEEE Conf. INFOCOM, pp.1-9, 2010. . 
[37] M. K. Han, A. Bhartia, L. Qiu, and E. Rozner, “O3: Optimized overlay-based opportunistic routing," in Proc. ACM Int. Symp.MobiHoc, May 2011.

[38] Y. Lin, B. Li, and B. Liang, "CodeOR: Opportunistic routing in wireless mesh networks with segmented network coding," in Proc. IEEE ICNP, pp. 13-22, 2008,

[39] Y. Lin, B. Liang, and B. Li, "SlideOR: Online opportunistic network coding in wireless mesh networks," in Proc. IEEE INFOCOM, 2010, pp. 1-5. D. Koutsonikolas, Y. Hu, and C. Wang, "XCOR: Synergistic interflow network coding and opportunistic routing," in Proc. ACM Annu. Int. Conf. MobiCom, pp. 1-3, Sep. 2008. .

[40] C. Westphal, "Opportunistic routing in dynamic ad hoc networks: The OPRAH protocol," in Proc. IEEE Int. Conf. MASS, pp. 570573, Oct. 2006. .

[41] Y. Yuan, H. Yang, S.Wong, S. Lu, andW. Arbaugh, "ROMER: Resilient opportunistic mesh routing for wireless mesh networks,” in Proc. IEEE Workshop WiMesh, pp.1-9, Sep. 2005.

[42] M. Nassr, J. Jun, S. Eidenbenz,A.Hansson, andA. Mielke, "Scalable and reliable sensor network routing: Performance study from field deployment," in Proc. 26th IEEE Conf. INFOCOM, pp. 670-678, May 2007.

[43] S. Guo, Y. Gu, B. Jiang, and T. He, "Opportunistic flooding in lowduty- cycle wireless sensor networks with unreliable links," in Proc. $15^{\text {th }}$ Annu. Int. Conf. MobiCom, pp. 133-144, 2009.

[44] M. Lu and J. Wu, "Opportunistic routing algebra and its applications, in Proc. IEEE INFOCOM, pp. 2374-2382, 2009.

[45] A. Vahdat and D. Becker, "Epidemic routing for partially-connected ad hoc networks," Technical Report CS 2000-06, Duke University, Durham, NC, USA, 2000.

[46] T. Spyropoulos, K. Psounis, and C. Raghavendra, "Spray and Wait: An efficient routing scheme for intermittently connected mobile networks, in Proc. ACM SIGCOMM WDTN, pp. 252-259, 2005.

[47] A. C. V. Erramilli, M. Crovella, and C. Diot, "Delegation forwarding,” in Proc. ACM Int. Symp. MobiHoc, pp. 251-259, May 2008.

[48] S. Nelson, M. Bakht, and R. Kravets, "Encounter-based routing in DTNs," in Proc. IEEE INFOCOM, pp. 846-854, Apr. 2009.

[49] C. Liu and J. Wu, "On multicopy opportunistic forwarding protocols in nondeterministic delay tolerant networks," IEEE Trans. Parallel Distrib.Syt., vol. 23, no. 6, pp. 1121-1128, Jun. 2012. .

[50] M. N. R. Bruno and M. Conti, "MaxOPP: A novel opportunistic routing for wireless mesh networks," in Proc. IEEE ISCC, pp. 255260, 2010.

[51] J. L. V. Conan and T. Friedman, "Fixed point opportunistic routing in delay tolerant networks," IEEE J. Sel. Areas Commun., vol. 26, no. 5, pp. 773-782, Jun. 2008.

[52] H. Dubois-Ferriere, M. Grossglauser, andM. Vetterli, "Valuable detours: Least-cost anypath routing," IEEE/ACM Trans. Netw., vol. 19, no. 2, pp. 333-346, Apr. 2011.

[53] R. P. Laufer, P. B. Velloso, L. F.M. Vieira, and L. Kleinrock, "PLASMA: A new routing paradigm for wireless multihop networks," in Proc. IEEE Conf. INFOCOM, pp. 2706-2710, 2012.

[54] R. Laufer, H. Dubois-Ferriere, and L. Kleinrock, "Multirate anypath routing in wireless mesh networks," in Proc. IEEE Conf. INFOCOM, 2009, pp. 37-45.

[55] M. K. Han, A. Bhartia, L. Qiu, and E. Rozner, “O3: Optimized overlay-based opportunistic routing,” in Proc. ACM Int. Symp.MobiHoc, May 2011, pp. 2:1-2:11.

[56] X. Zhang and B. Li, “Optimized multipath network coding in lossy wireless networks,” IEEE J. Sel. Areas Commun., vol. 27, no. 5, pp. 622-634, Jun. 2009.

[57] X. Fang, D. Yang, and G. Xue, “Consort: Node-constrained opportunistic routing in wireless mesh networks,” in Proc. IEEE Conf. INFOCOM 2011, pp. 1907-1915.

[58] Y. Li, A. Mohaisen, and Z. Zhang, “Trading optimality for scalability in large-scale opportunistic routing,” IEEE Trans. Veh. Technol., vol. 62, no. 5, pp. 2253-2263, Jun. 2013.

[59] A. Bhorkar, M. Naghshvar, T. Javidi, and B. Rao, “Adaptive opportunistic routing for wireless ad-hoc networks,” IEEE/ACM Trans. Netw., vol. 20, no. 1, pp. 243-256, Feb. 2012.

[60] R. Laufer, H. Dubois-Ferriere, and L. Kleinrock, "Polynomial-time algorithms for multirate anypath routing in wireless multihop networks,” IEEE/ACM Trans. Netw., vol. 20, no. 3, pp. 742-755, Jun. 2012. .

[61] X. Fang, D. Yang, and G. Xue, "MAP: Multi-constrained anypath routing in wireless mesh networks," IEEE Trans. Mobile Comput., vol. 12, no. 10, pp. 1893-1906, Oct. 2013

[62] X. Zhang and B. Li, "Dice: A game theoretic framework for wireless multipath network coding," in Proc. ACM Int. Symp. MobiHoc, May 2008, pp. 293-302.

[63] P. Tehrani, Q. Zhao, and T. Javidi, “Opportunistic routing under unknown stochastic models," in Proc. IEEE 5th Int. Workshop CAMSAP, 2013, pp. 145-148.

[64] H. Fussler, J. Widmer, M. Kasemann, M. Mauve, and H. Hartenstein, "Contention-based forwarding for mobile ad-hoc networks," Ad Hoc Netw., vol. 1, no. 4, pp. 351-369, Nov. 2003.

[65] B. Zhao, R. I. Seshadri, and M. C. Valenti, "Geographic random forwarding with hybrid-ARQ for ad hoc networks with rapid sleep cycles,” in Proc. IEEE GLOBECOM, Dec. 2004, pp. 3047-3052.

[66] K. Zeng,W. Lou, J. Yang, and D. Brown III, "On throughput efficiency of geographic opportunistic routing in multihop wireless networks,” Mobile Netw. Appl., vol. 12, no. 5, pp. 347-357, Dec. 2007.

[67] K. Zeng, W. Lou, and H. Zhai, "Capacity of opportunistic routing in multi-rate and multi-hop wireless networks," IEEE Trans. Wireless Commun., vol. 7, no. 12, pp. 5118-5128, Dec. 2008.

[68] Z. Zhao et al.,“Topology and link quality-aware geographical opportunistic routing in wireless ad-hoc networks,in Proc. IEEE $9^{\text {th }}$ IWCMC, Jul. 2013, pp. 1522-1527.

[69] D. Rosario et al"., "Assessment of a robust opportunistic routing for video transmission in dynamic topologies," in Proc. IFIP Wireless Days, 2013, pp. 1-6.

[70] R. Flury and R. Wattenhofer, "Randomized 3D geographic routing,” in Proc. IEEE Conf. INFOCOM, 2008, pp. 834-842.

[71] The Network Simulator -ns-2," http://www.isi.edu/nsnam/ns, 2013.

[72] J. Yoon, M. Liu, and B. Noble, “Random Waypoint Considered Harmful,” Proc. IEEE INFOCOM, pp. 1312-1321, 2003. 